\title{
On the discovery of magnon sidebands in insulating antiferromagnets
}

\author{
Robert M. White \\ Carnegie Mellon University, Pittsburgh, PA 15213, USA \\ E-mail: white@ece.cmu.edu \\ William M. Yen \\ Department of Physics and Astronomy, University of Georgia, Athens, GA 30602-2451, USA \\ E-mail: wyen@physast.uga.edu
}

Received February 4, 2005

\begin{abstract}
In this article, we reconstruct the course of events which led to the discovery and identification of magnon sidebands in the optical spectrum of simple antiferromagnetic insulators.
\end{abstract}

PACS: 75.30.Ds, 75.50.Ee

\section{Introduction}

In a recent article in which he summarizes his remarkable career and his many seminal contributions to our understanding of optical properties of the condensed phases [1], Don McClure writes:

«...But I was an unreconstructed academic and returned to academic life after receiving an offer from the University of Chicago in 1962. There, among other things, we built a pulsed magnet capable of producing fields of $20 \mathrm{~T}$ and saw the Zeeman effect in the triplet states of organic molecules.

More interesting though was the effect of these high fields on the absorption spectrum of $\mathrm{MnF}_{2}$ and similar transition metal compounds whose thermodynamic properties Willard Stout had been studying. We found that some of the spectra lines were split in the field as expected, but that others were not - a surprise which could be explained in terms of the antiferromagnetic structure of the crystal. While we were learning that there were such things as magnons as well as excitons in these crystals, a group of students of Art Schawlow at Stanford published a paper on the spin-wave sidebands (magnon side bands) of $\mathrm{MnF}_{2}$. We were in second place again but our paper nicely confirmed their interpretation. We never learned why they picked up $\mathrm{MnF}_{2}$ just when we $\operatorname{did} . . . \gg$

The paper McClure alludes to concerned the first observation of a magnon induced sideband in a magnetically ordered material [2]. The identification of these sidebands by a pair of young students and two post-docs illustrates how serendipity, a touch of good luck and reckless youthful enthusiasm often plays a role in scientific discovery. In this article, we describe the events that led to these developments in the hope that they will answer McClure's curiosity on this subject.

\section{The Stanford Hansen Microwave Laboratory}

The authors became acquainted with each other at Stanford in the Fall 1962; at that time, one of us (RMW) was in midst of his PhD thesis work in Marshal Sparks' theory group while the other (WMY) had just joined Art Schawlow's group in spectroscopy as a Research Associate. Schawlow had arrived earlier in the year at Stanford from Bell Labs and was assigned temporary lab space in the Hansen Microwave $\mathrm{Lab}$ and began establishing those programs which were later to be recognized with a Nobel Prize. The Microwave Lab was housed in a «temporary» building dating from the Second World War and the lab was extremely crowded; the forced contact in these close quarters led to considerable socialization between members of the six or seven different research groups utilizing Hansen and resulted in some beneficial interactions, as exemplified by our discovery of magnon sidebands.

Schawlow's interests at the time centered on the optical properties of various dopants in insulating solids which could be used for solid state lasers. Yen and other members of the group were given the task to un- 
derstand the thermal dependence of the widths and positions of the sharp line spectra of transition metals and rare earths in solids. These studies resulted in a number of publications in which the thermal broadening and shifts of the spectra were identified as arising from phonon-ion interactions. Of significance to this article, in Imbusch et al. [3], the isotope shifts of the $\mathrm{Cr}^{3+}$ R-lines in $\mathrm{Al}_{2} \mathrm{O}_{3}$ and $\mathrm{MgO}$ were related to changes in the phonon frequencies induced by the differences in the masses of the hosts and the reflection of these changes in the phonon sideband structure. In addition, in Yen et al. [4], observation of phonon sideband structure was first reported for a rare earth based material $\left({ }^{3} \mathrm{H}_{4}\right.$ to ${ }^{3} \mathrm{P}_{0}$ absorption of $\left.\mathrm{LaF}_{3}: \operatorname{Pr}^{3+}\right)$.

White's thesis involved extending Suhl's theory of spinwave instabilities in ferrites to antiferromagnets. Although the magnetic properties of antiferromagnets had been described by Néel in 1932, at that time they were more of a curiosity than a material with technological applications as were the ferrites. This is not the case today where antiferromagnets play a critical role in providing the giant magnetoresistance (GMR) so important in magnetic recording heads. White's thesis also explored the possibility of phonon instabilities which developed his appreciation for analogies between magnons and phonons.

\section{The Varian Labs and the Oasis}

The new Physics Building at Stanford, the Varian Building, was finished in 1963 and Schawlow's spectroscopy group moved over from Hansen. At that time, it was customary for a group of us Physics graduate students and post-docs to stop for a night cap at a local watering spot ( The Oasis» or affectionately «The $\mathrm{O}$ ») following our usual late evenings at the lab; there normally would be a whole table reserved to accommodate us and both authors were frequent attendees of these gatherings. The topic of conversation at them was wide ranging, even including Physics problems at times! On one of these gatherings, about the time the two publications mentioned above were submitted, a discussion on phonon induced sidebands arose during which White first raised the possibility of observing a magnon induced sideband; our discourse then shifted to the practicality of observing such a feature in magnetically ordered materials and on the appropriate materials which would allow the observation of these assisted transitions. The first requirement, of course, was that the material possess good optical properties and secondly that its crystalline and magnetic properties be well characterized. At that time there were no obvious ferromagnets with the desired optical properties.
Eventually, White settled on the antiferromagnet, $\mathrm{MnF}_{2}$, which was being investigated at Bell Labs from the resonance point of view and whose magnetic properties have been established. In fact, Vince Jaccarino once refered to $\mathrm{MnF}_{2}$ as the «fruit fly» of magnetics research. It is reasonably transparent and has a Néel temperature that is not too low. In addition, the room temperature spectra of the $\mathrm{Mn}^{2+}$ in $\mathrm{MnF}_{2}$ had been determined by Stout [5].

The «luck» we refered to above also had to do with selecting $\mathrm{MnF}_{2} . \mathrm{MnF}_{2}$ has the rutile crystal structure which does not have a center of symmetry between $\mathrm{Mn}$ pairs. It is these pairs that are excited in the sideband. This lack of symmetry enables the relatively strong electric dipole transition, without which this effect might not have been observable. This was subsequently pointed out by Tanabe et al. [6].

White actually purchased a sample of $\mathrm{MnF}_{2}$ using his advisor's theory funding to launch this exploration. Crystals and boules of $\mathrm{MnF}_{2}$ were also listed as available in a catalogue from SemiElements, a small company in Pennsylvania. We purchased a whole boule from this company; though we later obtained samples from Howard Guggenheim (Bell Labs) and from Bob Feigelson (Stanford), curiously, the samples derived from this boule proved to be the best samples we ever investigated. We never learned the origin of the boule as SemiElements went out of business soon after.

Samples in different orientations, $\sigma, \pi$, and $\alpha$ were cut from the boule and initial surveys of the absorption spectra were done at low temperature using a photographic B\&L spectrograph. Initial results were first reported at the 1963 APS Winter Meeting in Berkeley [7]; in that paper, we first reported sharp structure in the vicinity of the first two excited states of $\mathrm{Mn}^{2+}\left({ }^{4} T_{1 g}\right.$ and $\left.{ }^{4} T_{2 g}\right)$ and very strong absorptions in the ${ }^{4} A,{ }^{4} E$ region, but no clearly identifiable magnon-assisted transitions. The paper attracted very little attention but the preliminary results were encouraging enough to warrant continued interest on our part. Our activities attracted the attention of three members of the Schawlow group, initially Warner Scott and then Darrell Sell and Rick Greene; we began an earnest effort to obtain high resolution, low-temperature spectra of $\mathrm{MnF}_{2}$ using a scanning spectrometer and electronic detection in the winter of 1964 .

1964 was an exceptional active year for both the authors.

White had obtained an NSF postdoctoral fellowship and had joined C. Kittel's group at UC Berkeley. Again guided by analogy with phonon sidebands he developed a theory for the shape of the magnon sideband. This characteristic asymmetric lineshape be- 
came critical in the subsequent identification of the sideband in experimental data.

Yen, on the other hand, had begun a program to investigate optical energy transfer processes in rare earth activated systems and was in the process of interviewing for a permanent position; he was to accept an offer from Wisconsin-Madison in the Spring of 1965. We were able to revisit the $\mathrm{MnF}_{2}$ only occasionally during this period but fortunately both Darrell Sell and Rick Greene began to show an increased interest in this problem as 1964 progressed and it eventually became the subject of their $\mathrm{PhD}$ theses.

\section{Physics of Quantum Electronics Conference and the discovery}

These activities produced a small problem for Art Schawlow since none of his grants provided coverage for these investigations. He discussed these difficulties with Yen just prior to their departure for Puerto Rico to attend the Physics of Quantum Electronics Conference (PQEC, June 28-30, 1965); it was agreed during that discussion that the project would be transferred to Wisconsin when Yen assumed his faculty position there in the Fall of 1965 but that we would continue our investigations at Stanford until our return from the PQEC meeting. Schawlow also was aware that R.E. Dietz, L.F. Johnson and others at Bell Labs had been investigating the properties of magnetically ordered materials and suggested that Yen discuss our ideas with them to resolve any conflicts if any existed. Yen's paper on linewidth studies of energy transfer mechanisms [8] was scheduled in the same session as that of Dietz et al. which was entitled «Fluorescence from Magnetic Crystals» $\left(\mathrm{NiF}_{2}, \mathrm{CoF}_{2}, \mathrm{MnF}_{2}\right)$ [9]. During the question period, Bob Dietz was specifically asked by Yen whether he had observed any effects relatable to the collective magnetic excitations of the simple fluorides he investigated; before he could answer, Willi Lowe of the Hebrew University responded for him and empathetically stated that «magnetic interactions were notoriously weak and there was absolutely no chance they would couple to electronic interactions». Likely that at that point, Schawlow wrote off our effort as a lost cause.

As noted earlier, however, we had strong and tantalizing hints of sharp structure accompanying the lower ${ }^{4} T$ transitions of $\mathrm{Mn}^{2+}$ in $\mathrm{MnF}_{2}$; before his departure for PQEC, Yen, Sell and Greene mapped out a schedule of experimentation during his absence which included He $\lambda$-point temperature runs on a high resolution Jarrell-Ash scanning spectrograph with emphasis on the lowest ${ }^{4} T_{1 g}$ state of $\mathrm{MnF}_{2}$. Yen also scheduled himself for week of vacation in St. Thomas following the PQEC meeting to extend the time

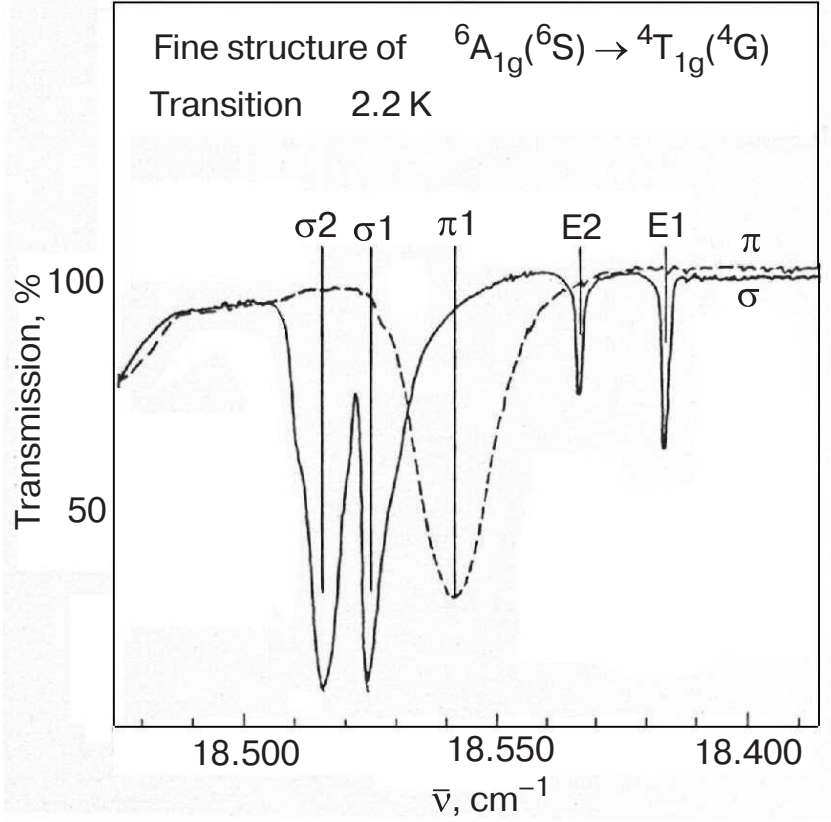

Fig. 1. Absorption spectrum of $\mathrm{MnF}_{2}$ in the range of $18.550 \mathrm{~cm}^{-1}$. The absorptions E1 and E2 are pure electronic transitions of $\mathrm{Mn}^{2+}$. The absorptions $\sigma 1, \sigma 2$, and $\pi 1$ are the first identified magnon-assisted transitions in a magnetic material.

before our efforts at Stanford were to be shut down. As it turns out, the additional week was not needed; Sell's lab book entry for the observation of what later to be definitely identified as magnon sidebands, shown in Fig. 1, was June 29, 1965, likely the very day of the session alluded to above! When these traces were shown to Schawlow in early July, he immediately understood the importance of the observation and shutting down the effort was, of course, never considered again. Schawlow's enthusiasm was also responsible for White returning to Stanford as an Assistant Professor in the fall of 1965 .

\section{Conclusions}

A complete description of the magnon sideband phenomenon was published in 1967 [10]. The fact that the peak of the sideband is directly related to the frequency of magnons at the Brillouin zone boundary enables one to study these modes quite easily, which previously could only be done by neutron scattering. At least half a dozen theses on various aspects of the spectra of magnetically ordered materials from Stanford and from Wisconsin followed in the following decades; this work contributed to a comprehensive understanding of the static and dynamic optical properties of ordered magnetic systems. These studies included studies on the luminescence properties of pure and doped $\mathrm{MnF}_{2}[11,12]$, the observation of induced photomagnetism [13], of magnon induced broadening 
of optical transitions [14], of circular and magnetic circular dichroism [15] and the discovery of biexciton annihilation in this material [16]. And recently, we have been able to identify sublattice splittings in $\mathrm{MnF}_{2}$ induced by dipolar interactions predicted earlier by one of us (RMW) [17,18].

It is interesting how often new concepts in science appear almost simultaneously in different contexts. While we were looking for what we now call an exciton-magnon excitation in $\mathrm{MnF}_{2}$ at Stanford, the very similar phenomenon of two-magnon excitation in the infrared was unknowingly being observed in $\mathrm{FeF}_{2}$ by I. Silvera at Berkeley [19] and later in $\mathrm{CoF}_{2}$ at Bell Labs [20]. In fact, the Berkeley IR absorption data with its anamolous peak at $154 \mathrm{~cm}^{-1}$ was presented in the very same session of the 1963th APS meeting in Berkeley at which we presented our first optical spectra of $\mathrm{MnF}_{2}$. And, as indicated by McClure's quote at the beginning of this article, his group was also studying the optical spectra of $\mathrm{MnF}_{2}$ and had been puzzled by the observation of transitions which did not split in a magnetic field [21]. Now that we understand this process we know that all these phenomenon are related, and their existence provides a very useful technique for studying magnon dynamics [22] as well as energy transfer in solids [23].

1. D.S. McClure, J. Lumin. 100, 47 (2002).

2. R.L. Greene, D.D. Sell, W.M. Yen, A.L. Schawlow, and R.M. White, Phys. Rev. Lett. 15, 656 (1965).

3. G.F. Imbusch, W.M. Yen, A.L. Schawlow, G.E. Devlin, and J.P. Remeika, Phys. Rev. 136, A481 (1964).

4. W.M. Yen, W.C. Scott, and A.L. Schawlow, Phys. Rev. 136, A271 (1964).

5. J.W. Stout, J. Chem. Phys. 31, 709 (1959).

6. Y. Tanabe, T. Moriya, and S. Sugano, Phys. Rev. Lett. 15, 1023 (1965).

7. W.M. Yen, W.C. Scott, and R.M. White, Bull. Am. Phys. Soc. II-9, 715 (1963).
8. W.M. Yen, R.L. Greene, and W.C. Scott, Optical Linewidth Studies Energy Transfer Mechanism Between Impurity Ions, in Proceedings, of the Physics of Quantum Electronics, P.L. Kelley, B. Lax, and P.E. Tannenwald (eds.), McGraw-Hill, New York (1966), p. 332.

9. R.E. Dietz, L.F. Johnson, and H.J. Guggenheim, Fluorescence of Magnetic Crystals, in Proceedings of the Physics of Quantum Electronics, P.L. Kelley, B. Lax, and P.E. Tannenwald (eds.), McGraw-Hill, New York (1966), p. 361.

10. D. Sell, R. Greene, and R.M. White, Phys. Rev. 158, 489 (1967).

11. R.L. Greene, D.D. Sell, R.S. Feigelson, G.F. Imbusch, and H.J. Guggenheim, Phys. Rev. 171, 600 (1968).

12. B.A. Wilson, W.M. Yen, J. Hegarty, and G.F. Imbusch, Phys. Rev. B19, 4238 (1978).

13. J.F. Holzrichter, M.L. Report no. 1912, Stanford University, November (1970).

14. M.W. Passow, D.L. Huber, and W.M. Yen, Phys. Rev. Lett. 23, 477 (1969).

15. Y.H. Wong, F.L. Scarpace, C.D. Pfeifer, and W.M. Yen, Phys. Rev. B9, 3086 (1974).

16. B.A. Wilson, J. Hegarty, and W.M. Yen, Phys. Rev. Lett. 41, 268 (1978).

17. D.J. Lockwood, R.M. White, and W.M. Yen, Phys. Rev. B69, 174413 (2004).

18. R.M. White, J. Appl. Phys. 36, 3653 (1965).

19. I. Silvera and M. Tinkham, Bull. Am. Phys. Soc. II-9, 714 (1963).

20. P.L. Richards, Bull. Am. Phys. Soc. II-10, 33 (1965).

21. P.G. Russell, D.S. McClure, and J.W. Stout, Phys. Rev. Lett. 16, 176 (1966).

22. L.D. Rotter, W.M. Dennis, and W.M. Yen, Phys. Rev. B42, 720 (1990).

23. G.P. Morgan and W.M. Yen, Optical Energy Transfer in Insulators, in Laser Spectroscopy of Solids, W.M. Yen (ed.), Springer Topics in Applied Physics, v. 65, Springer Verlag Berlin (1989), p. 77. 